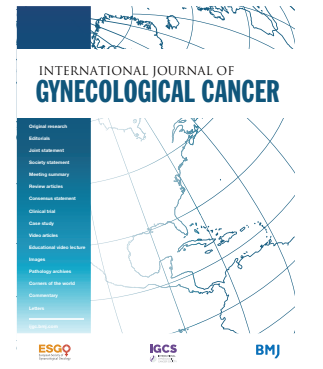

For numbered affiliations see end of article.

\section{Correspondence to}

Dr Francesca Moro, Dipartimento Scienze della Salute della Donna, del Bambino e di Sanità Pubblica, Fondazione Policlinico Universitario Agostino Gemelli, IRCCS, Rome 00168, Italy; morofrancy@gmail.com

Received 1 October 2020 Revised 8 0ctober 2020 Accepted 12 0ctober 2020 Published Online First 2 November 2020

\title{
Imaging modalities in fertility preservation in patients with gynecologic cancers
}

\author{
Francesca Moro (D) , ${ }^{1}$ Giulia Maria Bonanno, ${ }^{1}$ Benedetta Gui, ${ }^{2}$ Giovanni Scambia, ${ }^{1,3}$ \\ Antonia Carla Testa ${ }^{1,4}$
}

\section{ABSTRACT}

Fertility preservation is an integral component of clinical decision-making and treatment design. However, the selection criteria on imaging for patients eligible for fertility preservation is still unclear. The present review aimed to summarize the main findings reported in both the literature and international guidelines on the role of imaging in the selection of patients for fertility preservation. A search strategy was developed and applied to PubMed, Scopus, Web of Science, and EMBASE to identify previous citations reporting imaging and fertility preservation in patients with gynecological cancer. We also retrieved the published guidelines on the eligibility criteria for fertility-sparing treatment of gynecological neoplasms. A description of the internal multidisciplinary guidelines, clinically in use in our institution, is provided with representative clinical cases. The literature review revealed 1291 articles and 18 of these were selected for the analysis. Both ultrasound and MRI represented the primary imaging methods for selecting patients for fertility preservation in cervical and endometrial cancers. Eligibility criteria of fertilitysparing management in patients with cervical cancer were: tumor size $<2 \mathrm{~cm}$, tumor distance from the internal os $>1 \mathrm{~cm}$, and no parametrium invasion. For patients with endometrial cancer, these included no myometrial and cervical stroma invasion. Both ultrasound and MRI play a key role in characterizing adnexal masses. These modalities provide a useful tool in identifying small ovarian lesions, thus key in the surveillance of patients after fertility sparing surgery. However, efficacy in excluding disease beyond the ovary remains limited. This review provides an update of the literature and schematic outline for the counseling and management of patients with the desire for fertility preservation.

\section{INTRODUCTION}

Currently, there is an increasing tendency to treat women with cancer and ensure good quality of life, including future fertility. Indeed, fertility preservation is an integral component of clinical decision-making and treatment design. ${ }^{12}$ Fertility-sparing options for young patients with gynecologic cancer are influenced by patient age, type of primary cancer, treatment, and patient preferences. ${ }^{3}$ Defining which patients are eligible for fertility preservation significantly relies on accurate cancer staging. In this context, ultrasound evaluation ${ }^{4}$ and $\mathrm{MRI}^{5-7}$ play an important role in the preoperative assessment of gynecological cancers.
In the literature, however, there are currently few reviews on fertility-sparing treatment, ${ }^{4} 5$ 8-10 describing selection criteria on imaging for patients eligible for fertility preservation. ${ }^{71-14}$ An understanding of the potential pitfalls of imaging methods is crucial to avoid misinterpretation, as well as to guide management. The current review aims to summarize the main findings reported in both the literature and international guidelines (NCCN, ESMO/ESGO/ESTRO) on the role of imaging in the selection of patients eligible for fertility preservation. Additionally, a brief summary of the methodology used at our center for selecting these patients by using images of both ultrasound and MRI is described.

\section{METHODS}

\section{Review of the Literature}

A search strategy was developed and applied to PubMed, Scopus, Web of Science, and EMBASE to identify previous citations reporting imaging and fertility preservation in patients with gynecological cancer. We used the following words for selection: ("fertility preservation" or "fertility sparing" or "fertility-sparing" or "conservative surgery" or "conservative management" or "conservative treatment" or "trachelectomy" or "hysteroscopic endometrial resection" or "fertility-sparing management" or "fertility sparing management") and ("gynecological cancer" or "gynecology oncology" or "gynecologic malignancy" or "gynecologic cancer" or "gynecological malignancy" or "cervical cancer" or "cervical carcinoma" or "endometrial cancer" or "endometrial carcinoma" or "ovarian cancer" or "ovarian carcinoma" or "ovarian malignancy") and ("ultrasound" or "image" or "imaging" or "MRl" or "ultrasonography" or "magnetic resonance") and "imaging" or "ultrasound" and ("fertility preservation") and ("gynecological cancer") and "fertility sparing surgery" and ("gynecology" or "gynecological cancer") and ("fertility sparing") and ("imaging ") .

The search and selection criteria were restricted to full English language papers published from 1993 to 2020. We also retrieved the guidelines (NCCN, ESMO/ESGO/ESTRO) ${ }^{15-20}$ on the eligibility criteria for the fertility-sparing treatment of gynecological neoplasms. A description of the internal 


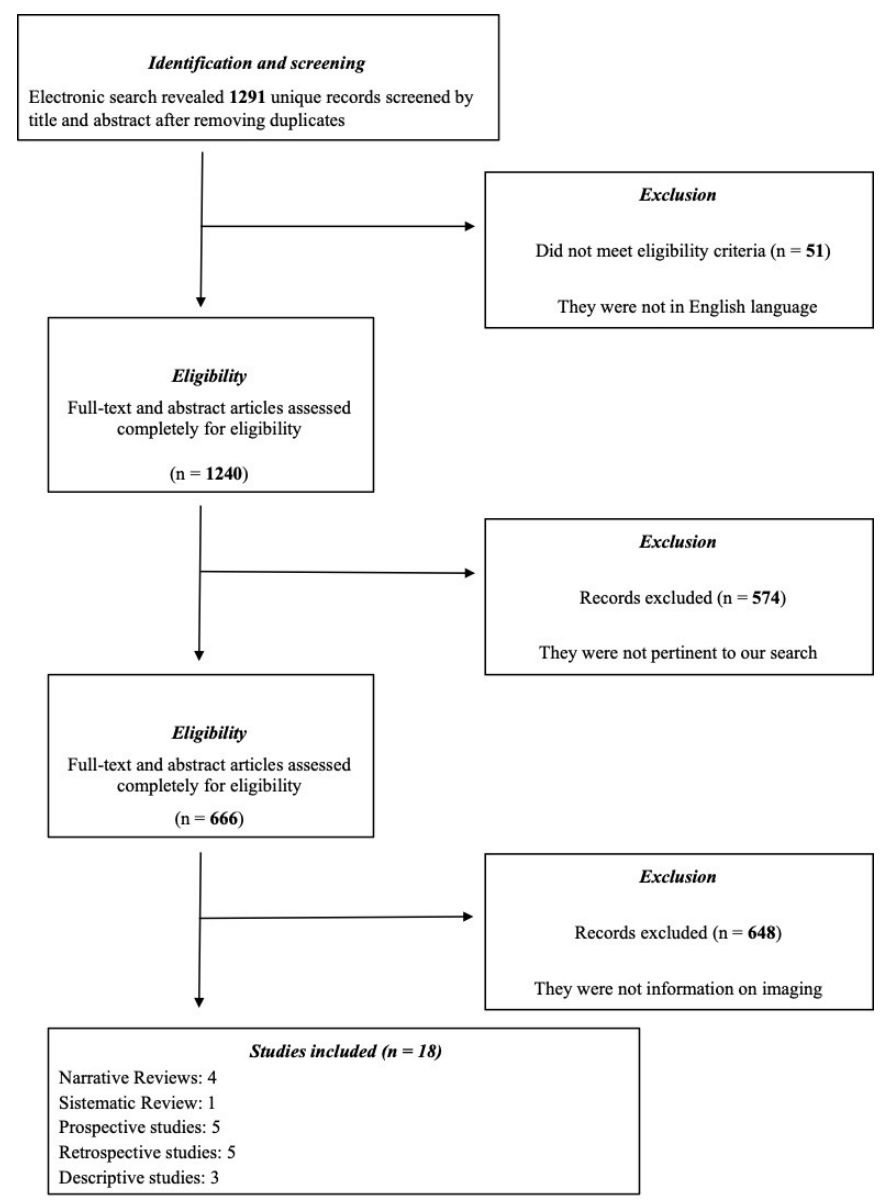

Figure 1 Flowchart diagram summarizing the selected articles.

multidisciplinary guidelines used in our institution is provided, with representative clinical cases.

\section{RESULTS}

The literature review revealed 1291 articles, and 51 of these were excluded as they were not in the English language. We also excluded 574 articles that were not pertinent to our search, and finally, excluded another 648 articles that were mainly related to fertility-sparing treatment, but with no information on imaging. On completion of all exclusion criteria, there were 18 remaining articles that were evaluated and reported herein. The flowchart diagram is shown in Figure 1. According to the primary site of disease, we divided the selected manuscripts into three main subgroups: cervical cancer, endometrial cancer, and ovarian cancer (Table 1).

\section{Cervical Cancer}

Cervical cancer is the second most commonly diagnosed cancer and the third cause of cancer death among women in less developed countries (sub-Saharan Africa, Latin America and the Caribbean, and Melanesia) with more than $40 \%$ of patients with early cervical cancer affected during reproductive age where there is a desire for future fertility. ${ }^{21}$ According to international society guidelines, including NCCN, ESMO/ESGO/ESTRO, patients with microinvasive disease (FIG0 stage IA1) and no lymphovascular space invasion (LVSI) are candidates for cone biopsy only. For patients with FIGO stage IA1 but with LVSI, the risk of node involvement is greater and a sentinel lymph node (SLN) mapping algorithm or pelvic lymphadenectomy is recommended. ${ }^{15}{ }^{16}$ Radical trachelectomy may offer a reasonable fertility-sparing treatment option for patients with cervical cancer of FIGO 2018 stage IA2 or IB1. Laparoscopic pelvic lymphadenectomy complements the procedure and can be performed with or without SLN mapping. ${ }^{15}{ }^{16}$ Patients with an histology of squamous cell carcinoma, adenocarcinoma, or adenosquamous carcinoma are eligible for fertility-sparing treatment, whereas patients with small cell neuroendocrine tumors, gastric type, or adenoma malignum are ineligible. ${ }^{15} 16$ Tumor size, tumor extending to the internal os, stromal invasion, parametrial invasion, and the presence of lymphovascular invasion and extrauterine spread are among the most important parameters when selecting patients who might benefit from fertility-sparing surgery.

Among the articles on cervical cancer and fertility-sparing options, we selected 11 articles dealing with MRI and one with ultrasound evaluation. In particular, we found one systematic review, ${ }^{10}$ one narrative review, ${ }^{8}$ three retrospective studies, ${ }^{11-13}$ two prospective studies, ${ }^{22}{ }^{23}$ and one descriptive article on MRI in cervical cancer, ${ }^{14}$ whereas four articles (two descriptive studies ${ }^{67}$ and two narrative reviews ${ }^{4}$ ) reported fertility-sparing in several gynecological cancers including data for fertility preservation in patients with cervical cancer (three on MRI and one on ultrasound) (Table 1).

\section{Tumor Size}

The current selection criteria for vaginal radical trachelectomy include a tumor size of $<2 \mathrm{~cm}$ where the measurement is based on the three dimensions (anteroposterior, craniocaudal, and transverse). ${ }^{5-811121423} \mathrm{MRI}$ has been shown to be accurate in evaluating tumor size to within $5 \mathrm{~mm}$ of surgical dimension. ${ }^{14}$ The shape of the lesion and its pattern of growth, which may be exophytic (outward or eccentric to the cervical canal) or endophytic (inward or along the cervical canal), must be reported. ${ }^{14}$ For tumors measuring more than $2 \mathrm{~cm}$, neoadjuvant chemotherapy followed by conization or trachelectomy may also be an acceptable choice, but downstaging by neoadjuvant chemotherapy in patients with FIGO 2009 Stage IB1 and IB2 before fertility-sparing surgery is still an experimental technique. $^{15}$

\section{Tumor-to-Internal 0s Distance}

The ideal distance between the superior margin of the cervical tumor and the internal os (inner aspect of the cervix) is $\geq 1 \mathrm{~cm}$ in order to allow for a $1 \mathrm{~cm}$ of healthy cervical stroma between internal os and anastomosis, however, some authors accept $\geq 0.5 \mathrm{~cm}^{5-71012-142223}$ The internal os is defined as the region where the uterine vessels enter the uterus and the narrowing of the uterine contour where the low-signal-intensity cervical stroma changes to the intermediatesignal intensity of the uterine myometrium. The distance between the internal os and the cranial margin of the tumor should be assessed in the sagittal plane.$^{12}$ MRI has a high specificity for identifying invasion of uterine cervical cancer into the internal os. ${ }^{1013}$ The length of the endocervical canal is also an important measurement for adequate surgical planning. ${ }^{14}$ Indeed, it was suggested that a cervix length of more than $2 \mathrm{~cm}$ is necessary for performing trachelectomy or conization. 
Table 1 Summary of studies reporting imaging parameters indicating patients eligible for fertility preservation

\begin{tabular}{|c|c|c|c|c|c|}
\hline Author, year & Pathology & Study design & Imaging & Participants & Selected parameters for FS \\
\hline $\begin{array}{l}\text { Peppercorn PD } \\
1999\end{array}$ & Cervical cancer & Prospective study & $\mathrm{MRI}$ & 30 & $\begin{array}{l}\text { Tumorous distance from the internal os (sensitivity } 100 \% \\
\text { and specificity } 96 \% \text { ) }\end{array}$ \\
\hline Rob L et $\mathrm{al}^{8} 2010$ & Cervical cancer & Narrative review & MRI & NA & Tumor size <2 cm; cervical stroma infiltration $(<50 \%)$ \\
\hline $\begin{array}{l}\text { Lakhman } Y \text { et al } \\
2012\end{array}$ & Cervical cancer & Retrospective study & $\mathrm{MRI}$ & 62 & $\begin{array}{l}\text { Tumor size } \leq 2 \mathrm{~cm} \text {, absence of deep cervical stromal } \\
\text { invasion, tumorous distance from the internal os }>5 \mathrm{~mm}\end{array}$ \\
\hline $\begin{array}{l}\text { De Boera } P \text { et } \text { al }^{10} \\
2013\end{array}$ & Cervical cancer & Systematic review & MRI & 366 & Internal os involvement (sensitivity $91 \%$, specificity $97 \%$ ) \\
\hline Noël P et al ${ }^{14} 2014$ & Cervical cancer & Descriptive study & MRI & NA & $\begin{array}{l}\text { Tumor size } \leq 2 \mathrm{~cm} \text {; stromal invasion }<50 \% \text { or } 1 \mathrm{~cm} \text {; } \\
\text { tumorous distance from the internal os } \geq 1 \mathrm{~cm} \text {; no lymph } \\
\text { nodes; no extension beyond the cervix; cervix length } \\
\geq 2 \mathrm{~cm}\end{array}$ \\
\hline $\begin{array}{l}\text { Downey } \mathrm{K} \text { et al } \\
2014\end{array}$ & Cervical cancer & Prospective study & $\begin{array}{l}\text { MRI } \\
\text { (endovaginal) }\end{array}$ & 55 & Tumor size; tumorous distance from the internal os \\
\hline Joumelle $C^{11} 2016$ & Cervical cancer & Retrospective study & MRI & 21 & $\begin{array}{l}\text { Tumor size } \leq 2 \mathrm{~cm} \text { (visible in } 100 \% \text { of Stage } 1 \mathrm{~B} 1 \text { patients, } \\
\text { in } 60 \% \text { of Stage } 1 \mathrm{~A} 2 \text { patients), to be strictly intracervical, } \\
\text { no parametrium invasion }\end{array}$ \\
\hline $\begin{array}{l}\text { Bhosalea PR et } \text { al }^{13} \\
2016\end{array}$ & Cervical cancer & Retrospective study & MRI & 79 & $\begin{array}{l}\text { Tumorous distance from the internal os (specificity } \\
93.8 \% \text { ); assessment of } \geq 5 \mathrm{~mm} \text { distance from the } 1 \mathrm{O} \\
\text { (specificity of } 98.3 \% \text { ) }\end{array}$ \\
\hline Suh DS et $\mathrm{al}^{29} 2009$ & $\begin{array}{l}\text { Endometrial } \\
\text { cancer }\end{array}$ & Retrospective study & MRI & 301 & $\begin{array}{l}\text { Myometrial invasion absent (sensitivity } 68.8 \% \text {, specificity } \\
74.4 \% \text {, PPV } 86.9 \% \text {, NPV } 49.2 \% \text { ) }\end{array}$ \\
\hline Cade TJ et $\mathrm{al}^{28} 2010$ & $\begin{array}{l}\text { Endometrial } \\
\text { cancer }\end{array}$ & Retrospective study & MRI & 111 & $\begin{array}{l}\text { Myometrial invasion absent (PPV for all grades } 87 \% \text { and } \\
95 \% \text { for grade, NPV } 35 \% \text { for all grades and } 46 \% \text { for } \\
\text { grade 1) }\end{array}$ \\
\hline $\begin{array}{l}\text { Rodolakis A et } \text { al }^{9} \\
2014\end{array}$ & $\begin{array}{l}\text { Endometrial } \\
\text { cancer }\end{array}$ & Narrative review & $\begin{array}{l}\text { MRI and } \\
\text { ultrasound }\end{array}$ & NA & $\begin{array}{l}\text { Myometrial invasion absent; no synchronous or metastatic } \\
\text { ovarian tumors }\end{array}$ \\
\hline $\begin{array}{l}\text { Sakane } \mathrm{M} \text { et } \mathrm{al}^{30} \\
2017\end{array}$ & $\begin{array}{l}\text { Endometrial } \\
\text { cancer }\end{array}$ & Prospective study & $\mathrm{MRI}$ & 26 & $\begin{array}{l}\text { Myometrial invasion absent (sensitivity } 42 \% \text {, specificity } \\
85 \%, \text { PPV } 79 \% \text {, NPV 47\%) }\end{array}$ \\
\hline $\begin{array}{l}\text { Franchi D et } \text { al }^{39} \\
2016\end{array}$ & $\begin{array}{l}\text { Borderline } \\
\text { ovarian } \\
\text { recurrence }\end{array}$ & Prospective study & Ultrasound & 34 & $\begin{array}{l}\text { Growth rate of borderline recurrence ranges from a } \\
\text { minimum of } 0.06 \mathrm{~mm} / \mathrm{months} \text { for cysts }<10 \mathrm{~mm} \text { up to } 1.92 \\
\mathrm{~mm} / \mathrm{months} \text { for cysts }>20 \mathrm{~mm}\end{array}$ \\
\hline $\begin{array}{l}\text { Jones BP et } \mathrm{al}^{40} \\
2017\end{array}$ & $\begin{array}{l}\text { Borderline } \\
\text { ovarian } \\
\text { recurrence }\end{array}$ & Prospective study & $\begin{array}{l}\text { Intraoperative } \\
\text { ultrasound }\end{array}$ & 7 & $\begin{array}{l}\text { Intraoperative US to facilitate complete tumor excision in } \\
\text { recurrent SBOT minimizing the removal of ovarian tissue }\end{array}$ \\
\hline Fischerova D 2011 & $\begin{array}{l}\text { Cervical cancer, } \\
\text { ovarian cancer }\end{array}$ & Narrative review & Ultrasound & NA & $\begin{array}{l}\text { Cervical cancer: tumorous distance from the internal } \\
\text { os }>1 \mathrm{~cm} \text {. Ovarian tumors: no tumor capsula invasion, } \\
\text { controlateral ovary normal. }\end{array}$ \\
\hline $\begin{array}{l}\text { Rockall AG et al } \\
2016\end{array}$ & $\begin{array}{l}\text { Cervical cancer, } \\
\text { endometrial } \\
\text { cancer, ovarian } \\
\text { cancer }\end{array}$ & Descriptive study & $\begin{array}{l}\text { MRI, } \\
\text { ultrasound }\end{array}$ & NA & $\begin{array}{l}\text { Cervical cancer: tumor size } \leq 2 \mathrm{~cm} \text { (or } \leq 2.5 \mathrm{~cm} \text { if the lesion } \\
\text { is exophytic); tumorous distance from the internal os } \\
>1 \mathrm{~cm} \text {; no parametrium invasion; no nodal enlargement; } \\
\text { no pelvic metastases. Ovarian cancer: no indication for } \\
\text { FS-only characterization of adnexal masses. Endometrial } \\
\text { cancer: no cervical and myometrial invasion }\end{array}$ \\
\hline McEvoy et $\mathrm{al}^{5} 2017$ & $\begin{array}{l}\text { Cervical cancer, } \\
\text { endometrial } \\
\text { cancer, ovarian } \\
\text { cancer }\end{array}$ & Narrative review & MRI & NA & $\begin{array}{l}\text { Cervical cancer: tumor size } \leq 2 \mathrm{~cm} \text { for fertility preservation } \\
\text { (some centers accept tumor size } \leq 4 \mathrm{~cm} \text {, particularly if } \\
\text { exophytic); tumor-to-internal os distance } \geq 1 \mathrm{~cm} \text { (some } \\
\text { centers accept } \geq 0.5 \mathrm{~cm} \text { ); cervical stromal invasion } \\
\text { (CSI) }<50 \% \text { (some centers accept: any degree of CSI); } \\
\text { absence of parametrial invasion; absence of lymph } \\
\text { node metastases. Endometrial cancer: disease confined } \\
\text { to the endometrium; absence of any myometrial } \\
\text { invasion; absence of cervical stromal invasion; absence } \\
\text { of synchronous primary ovarian cancer or ovarian } \\
\text { metastases; absence of lymph node metastases. Ovarian } \\
\text { tumors: no indication for FS-only to discriminate between } \\
\text { benign and malignant tumors }\end{array}$ \\
\hline Stein EB ${ }^{6} 2019$ & $\begin{array}{l}\text { Cervical cancer, } \\
\text { endometrial } \\
\text { cancer, ovarian } \\
\text { cancer }\end{array}$ & Descriptive study & MRI & NA & $\begin{array}{l}\text { Cervical cancer: tumor size } \leq 2 \mathrm{~cm} \text {; tumorous distance } \\
\text { from the internal os } \geq 1 \mathrm{~cm} \text {; cervical stromal invasion } \\
<50 \% \text {; nodal or distant metastatic disease absent. } \\
\text { Endometrial cancer: no myometrial and cervical invasion; } \\
\text { no extrauterine disease. Ovarian cancer: no indication for } \\
\text { FS-only characterization of adnexal masses; absence of } \\
\text { extraovarian disease }\end{array}$ \\
\hline
\end{tabular}


Depth of Stromal Cervical Invasion

To achieve a fertility-sparing treatment, stromal cervical invasion should be less than half of the stromal thickness. ${ }^{5} 681214$ The assessment of stromal invasion at MRI has been demonstrated to be imprecise.

Stromal invasion is seen on T2-weighted MRI as partial or complete disruption of the low-signal-intensity cervical stroma by an intermediate- to high-signal-intensity lesion. ${ }^{14}$ The presence of a low-signal-intensity ring of cervical stroma around the tumor excludes parametrial involvement with a negative predictive value of $94 \%-100 \%{ }^{14}$ The presence of a complete disruption of the hypointense stromal rim has been shown to have only a $50 \%$ predictive value for parametrial involvement. However, if the hypointense cervical stromal ring is completely interrupted by the tumor, either focally or diffusely, with the presence of nodular or spiculated tumor-to-parametrial interface, or encasement of periuterine vessels, the accuracy for parametrial involvement raises to $77 \%-96 \%$. ${ }^{14}$ Tumor spread beyond the cervix is a contraindication to conservative surgery. ${ }^{5711}$

\section{Lymph Node or Distant Metastases}

Lymph node involvement is also a contraindication to fertilitypreserving surgery. ${ }^{5-7}{ }^{14}$ Regarding ultrasound examination, one narrative review describing ultrasound parameters for fertility preservation for gynecological cancer reported data on cervical cancer. ${ }^{4}$ According to this manuscript, ultrasound examination provides detailed images of the cervical tumor, and tumorous distance from the internal os. Moreover, with ultrasound examination, deep stromal tumor invasion, parametria, and metastatic disease (bladder infiltration, rectum infiltration) can be evaluated. ${ }^{4}$ Ultrasound has demonstrated low sensitivity and high specificity in evaluating lymph node metastases in cervical cancer. ${ }^{24}$ In our institution, preoperative evaluation of patients eligible for fertility preservation is based on both ultrasound and MRI imaging. We acknowledge that, according to international guidelines, in patients with early cervical cancer, transvaginal ultrasound examination is sufficient if performed by experienced ultrasound examiners, as it is able to provide fundamental parameters for the selection of patients for fertility preservation. ${ }^{1516}$ However, despite the high costs, in our institution we also request MRI in order to have a panoramic assessment of the pelvis, with particular regards to lymph node evaluation. On the other hand, we do not exclude transvaginal examination as it represents the first diagnostic approach in all our oncological patients.

\section{Ultrasound Methodology}

Patients are examined in the lithotomy position with an empty bladder with a transvaginal or transrectal approach: this is complemented by an abdominal ultrasound examination to evaluate for lymph node enlargement within the pelvis. ${ }^{24}$ Cervical tumor is measured (in $\mathrm{mm}$ ) in three dimensions: cervical fundal diameter, anteroposterior diameter in sagittal projection, and lateral diameter in transverse plane. It usually appears as a solid lesion with mostly hypoechoic echostructure in comparison with the surrounding cervical stroma. ${ }^{25}$ Echogenicity of the tumor can appear as isoechoic, hyperechoic, or mixed, according to the surrounding cervical stroma (Figure 2). ${ }^{24}$ According to its growth pattern, the
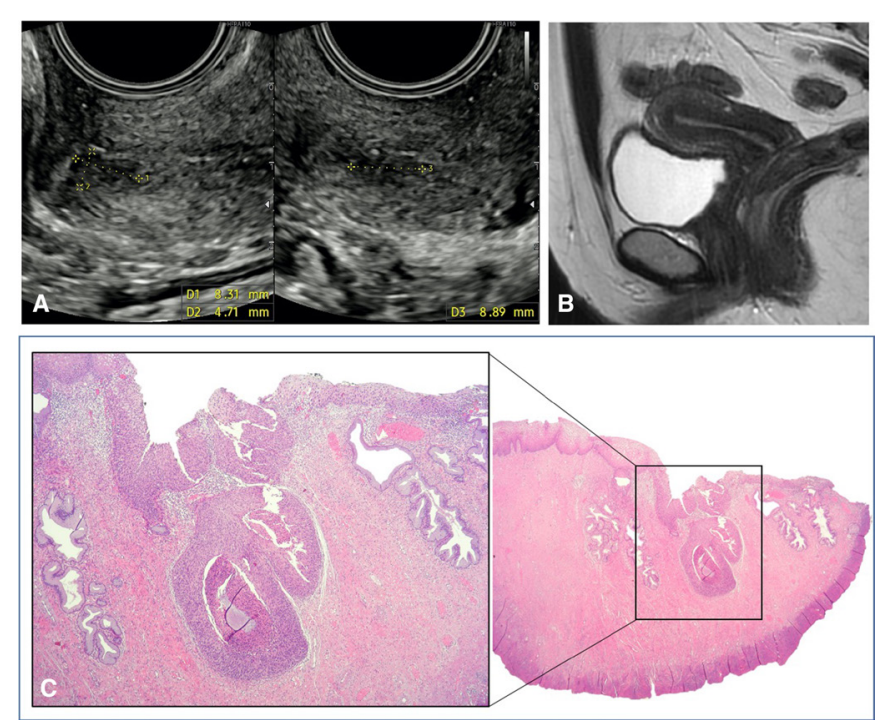

Figure 2 Ultrasound, MRI, and microscopic images of a 33 years' old patient with abnormal uterine bleeding. Ultrasound image (a) shows a hypoechoic lesion of $8 \times 4 \times 8 \mathrm{~mm}$ in size, with irregular margins suspicious for an early cervical cancer. The sagittal T2-weighted Mr image (b) shows the normal appearance of the cervix, with no visible tumor; no area of restricted diffusion at diffusion-weighted MR imaging or abnormal contrast enhancement was present (not shown). Histology (c) confirmed an area of $6 \mathrm{~mm}$ (zoom area in the box) of severe cervical dysplasia.

cancer may appear as a 'mushroom-shaped' lesion when exophytic and 'ovoidal' or 'conic-shaped' lesions when endophytic. ${ }^{25}$

The next step is the evaluation of the superior margin of the tumor to internal os distance. The total length of the cervix is also evaluated (Figure 3). The third priority is to evaluate the extent of tumor stromal invasion. The proportion of stromal invasion can be defined as either partial $(\leq 2 / 3$ or $>2 / 3$ ) or full thickness or, more precisely, it can be defined based on the measurement of tumorfree stroma. ${ }^{4}$ One should then evaluate parametrial involvement, at the level of anterior, lateral, and dorsal parametria. ${ }^{2526}$

\section{MRI Protocol and Methodology}

MRI protocol. At our institution, patients are examined with a 1.5T MR scanner using a standard 8-channel phased-array body coil. Patients are encouraged to fast 4 hours and empty the bladder prior to MRI examination to reduce peristalsis-related motion artifacts. An anti-peristaltic agent is administered intramuscularly at the start of the examination to further reduce peristalsis. Conventional sequences included axial T1-weighted and T2-weighted sequences for panoramic pelvic evaluation and high-resolution T2-weighted sequences performed in the sagittal, axial oblique (perpendicular), and coronal oblique planes (parallel) along the long axis of the cervix. Diffusion-weighted imaging is performed in the sagittal and axial oblique planes in the same orientation as T2-wieghted planes $\left(b=0\right.$ and $800-1000 \mathrm{sec} / \mathrm{mm}^{2}$ ) and apparent diffusion coefficient maps obtained. Large field-of-view T2-weighted (T2WI) images from the top of the kidneys to the iliac crests are acquired to exclude hydronephrosis and para-aortic lymphadenopathy. Dynamic contrast-enhanced MRI (DCE-MRI) is currently optional with newly diagnosed cervical cancer at an advanced stage. 

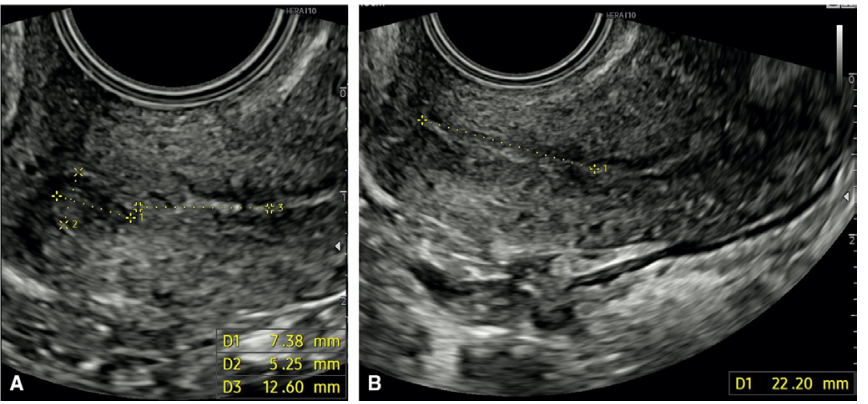

Figure 3 Ultrasound images showing the distance between the superior margin of the cervical lesion and the internal uterine os (a) and the total cervix length (b).

However, DCE-MRI has been demonstrated to improve tumor-tocervical stromal contrast in patients with small tumors $(<2 \mathrm{~cm})$ and should be used in patients considered for fertility preservation. ${ }^{14}$ We use sagittal DCE-MRI both before and after administration of contrast material, in order to identify small-volume lesions that may be difficult to detect on T2-weighted images and to better evaluate stromal invasion. Diffusion-weighted imaging should always be read in combination with T2WI sequences and contrast-enhanced images.

Cervical cancer demonstrates intermediate singal intensity on T2-weighted imaging, high-signal intensity on high b-value diffusion-weighted imaging,and low- signal intensity on the apparent diffusion coefficient map.

MRI reports to assess eligibility for fertility-sparing treatment should include (Figure 4):

i. Uterus information: length of the cervix from the internal os to the external os and length of the endometrial cavity from the fundus to the internal os, both measured on sagittal plane.

ii. Tumor information: diameter of the tumor on three axes, evaluated on sagittal plane for cranio-caudal and anteroposterior diameters and on oblique axial for transvers diameter; site of the tumor within the cervix (anterior or posterior lip, right or
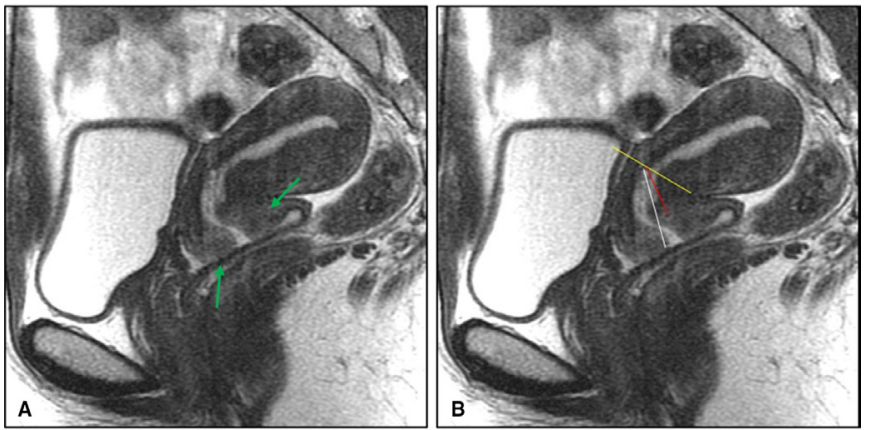

Figure 4 A sagittal T2-weighted MR image (a) showing soft tissue with intermediate signal intensity (green arrows), consistent with a cervical tumor, in the anterior and posterior lips of the cervix with maximum diameter of $2 \mathrm{~cm}$, with a distance from the tumor and the internal uterine os more than $1 \mathrm{~cm}$. sagittal T2-weighted MR image. (b) shows the measurements that should be assessed in order to plan fertility-sparing treatment. level of internal uterine os (yellow line). Length of the cervix from the internal uterine os to the external uterine os (white line), distance from the cranial margin of the tumor to the internal os (red line). left side, circumferential); tumor growth characteristics (exophytic or infiltrating); presence and extent of cervical stroma infiltration $(<50 \%$ or $1 \mathrm{~cm}$ with measurement of free stromal ring; or full-thickness); and distance of the proximal margin of the tumor to the internal os, vaginal fornix, or parametrium

iii. Lymph nodes information: absence or presence of pelvic or para-aortic nodal enlargement (short-axis diameter larger than $10 \mathrm{~mm}$ ); and other suspicious lymph nodes characteristics consist of round morphology, irregular borders and presence of necrosis.

iv. Other findings in pelvis or abdomen: presence of peritoneal implants, bladder, rectum, ovaries, liver, kidney, bone.

\section{Endometrial Cancer}

Endometrial cancer is the most common gynecological cancer in developed countries. More than $90 \%$ of endometrial cancers occur in women $>50$ years of age, with a median age at diagnosis of 63 years. However, $4 \%$ of patients diagnosed with endometrial cancer are younger than 40 years, and many of these patients wish to conceive. ${ }^{27}$ According to international societies and guidelines, including NCCN, ESMO/ESGO/ESTRO ${ }^{17}{ }^{18}$ patients with an histology of endometrioid adenocarcinoma, grade 1or 2, or a premalignant condition (atypical hyperplasia), are eligible for fertility presentation, whereas patients with other histologies (serous carcinoma, clear cell carcinoma, carcinosarcoma) are ineligible. ${ }^{717} 18$

Among the literature on endometrial cancer and fertility-sparing, we selected three on MRI and one on MRI and ultrasound evaluation. In particular, we found three retrospective studies ${ }^{28-30}$ on MRI and endometrial cancer and one narrative review on MRI and ultrasound; ${ }^{31}$ and three articles (two descriptive studies ${ }^{67}$ and one narrative review ${ }^{5}$ ) reported fertility-sparing and gynecological cancer including data on MRI parameters for fertility preservation in endometrial cancer patients (Table 1). Myometrial and cervical stromal invasion: absence of myometrial and cervical invasion is required to consider fertility-sparing treatment. ${ }^{5-7}{ }^{28-31} \mathrm{MRI}$ has high sensitivity in diagnosing myometrial invasion. ${ }^{28-31}$ However, very early myometrial invasion may be difficult to identify by MRI. ${ }^{7}$ Evidence of an uninterrupted low-signal-intensity junctional zone on T2WI is useful to exclude myometrial invasion and to confirm that tumor is confined to the endometrial canal. ${ }^{6}$ On DCE-MRI, the presence of a continuous rim of subendometrial enhancement of the inner junctional zone, evident within the first minute after contrast administration, helps to exclude myometrial infiltration. On T2WI, cervical stromal invasion is suspected when intermediate-to-high-signal-intensity tumor disrupts low-signal intensity cervical stroma. ${ }^{5}$ On DCE-MRI, stromal invasion is suspected when a hypo-enhancing tumor disrupts continuous enhancement of cervical epithelium. ${ }^{5}$

\section{Absence of Synchronous Primary Ovarian Cancer or Ovarian Metastases}

Synchronous or metastatic ovarian cancer in women with endometrial cancer is relatively uncommon, but this has to be excluded when considering fertility-sparing treatment. ${ }^{5-7} 31$ The presence of a large endometrial tumor and a small ovarian lesion may favor diagnosis of ovarian metastasis from endometrial cancer, whereas a small endometrial tumor and a large unilateral complex ovarian mass \pm endometriosis raises the possibility of a synchronous primary ovarian malignancy. ${ }^{5}$ 

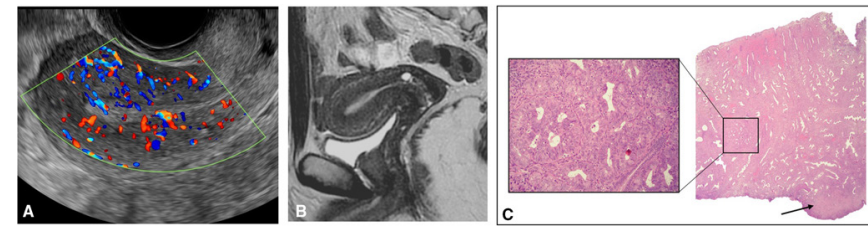

Figure 5 Ultrasound, MRI and microscopic images of a 36 years' old patient with histological diagnosis of endometrial adenocarcinoma of grade 1 (histology obtained from previous polypectomy). Color Doppler image (a) shows an endometrium with homogeneous echostructure, no myometrial invasion, and a single dominant vessel penetrating into the endometrial tissue. sagittal T2-weighted MR image. (b) shows normal thickness endometrium with no evidence of myometrial invasion. No area of restricted diffusion at diffusion-weighted MR imaging or abnormal sub-endometrial contrast enhancement was observed within the inner myometrium (not shown). The patient underwent hysteroscopic ablation of the endometrium. Final histology (c) confirmed adenocarcinoma of grade 1 (zoom) with no myometrial invasion (black arrow).

Regarding ultrasound examination, one narrative review has been published, describing ultrasound parameters for fertility preservation on endometrial cancer. ${ }^{31}$ Transvaginal ultrasound examination showed comparable accuracy to MRI scan and could be used in centers with difficult access to an MRI scan to define myometrial invasion and exclude suspicious ovarian lesions. ${ }^{31}$

\section{Ultrasound Methodology}

In our institution, preoperative evaluation of patients eligible for fertility preservation treatment is mainly based on transvaginal ultrasound examination or alternatively on MRI.

Endometrial lesions are described at ultrasound according to International Endometrial Tumor Analysis (IETA) terminology. ${ }^{32}$

Endometrial tumor is measured in three orthogonal diameters: anteroposterior (tumor thickness) and craniocaudal (tumor length) diameters, in the sagittal plane, and laterolateral diameter (tumor width), in the transverse plane. ${ }^{33}$ Color or power Doppler is used to assess the vascular pattern. Indeed, endometrial tumors with poor vascularization and with a "single vessel" patter are more indicative of a tumor of grade 1 and Stage IA than tumors with rich vascularization and multiple vessel patter that are more indicative of tumors at advanced stage. Moreover, one should measure the tumor-free margin in the plane in which one observes the smallest distance from the tumor to the serosa. ${ }^{34}$

The depth of myometrial invasion is evaluated subjectively and classified into no invasion, $<50 \%$, or $\geq 50 \%$ (Figure 5). Invasion of the cervix is diagnosed when the neoplastic tissue extends caudally merging with the endocervical mucosa. It is important to apply slight pressure with the transvaginal probe, in order to differentiate between mild protrusion of the endometrial tissue to the cervical canal and true infiltration of the endocervical mucosa. ${ }^{34}{ }^{35}$ Finally, assessment of the adnexa is suggested to exclude adnexal masses.

\section{MRI Protocol and Methodology}

MRI protocol for the evaluation of a patient with endometrial cancer is similar to the previous one described for cervical cancer but with some differences. High-resolution T2-weighted sequences performed in the axial oblique and coronal oblique planes are respectively obtained perpendicular and parallel to endometrial cavity. Diffusion-weighted imaging is also performed in the sagittal and axial oblique planes in the same orientation as T2-wieghted planes ( $\mathrm{b}=0$ and $800-1000 \mathrm{sec} /$ $\mathrm{mm}^{2}$ ) and apparent diffusion coefficient Imaps obtained. DCE-MRI is essential for local staging of endometrial cancer because it is helpful in delineating myometrial invasion. DCE-MRI is acquired in the sagittal plane with delayed contrast-enhanced images acquired in the axial oblique plane (90-120s after intravenous administration of gadolinium). Diffusion-weighted imaging should always be read in combination with $\mathrm{T} 2 \mathrm{Wl}$ sequences and contrast-enhanced images.

Endometrial cancer shows similar signal intensity on T2WI to the myometrium and relative hyperintensity to the adjacent low-signal junctional zone (inner myometrium). Most tumors enhance more slowly and less intensely than adjacent myometrium. Maximal tumorto-myometrial contrast occurs at delayed phase after intravenous administration of gadolinium with DCE-MRI. Endometrial tumors show high-signal intensity on the high-b-value diffusion-weighted imaging and low signal on apparent diffusion coefficient maps. However, the normal endometrium also shows slight high signal on diffusionweighted imaging due to its cellularity, but minor restriction compared with that of endometrial cancer.

MRI reports to assess eligibility for fertility-sparing treatment include:

i. Tumor information: diameter of the tumor on three axes, evaluated on sagittal plane for cranio-caudal (tumor length) and anteroposterior (tumor thickness) diameters, and on oblique axial for transvers diameter (tumor width); site of tumor within the endometrial cavity (corpus, fundus, anterior, or posterior wall; right or left side, cornus); absence or presence of myometrial invasion (<0r $>50 \%)$; and absence or presence of cervical stromal invasion (Figure 5).

ii. Ovarian information: evidence of synchronous primary ovarian cancer or ovarian metastases

iii. Lymph nodes information: absence or presence of pelvic or para-aortic nodal enlargement (short-axis diameter larger than $10 \mathrm{~mm}$ ); and other suspicious lymph nodes characteristics consist of round morphology, irregular borders, and presence of necrosis.

iv. Other findings in pelvis or abdomen: bladder, rectum, ovaries, liver, kidney, presence of peritoneal implants, bone.

\section{Ovarian Cancer}

Ovarian cancer is the leading cause of death among all gynecological cancers in developed countries, with most patients presenting with advanced stage. More than $90 \%$ of malignant ovarian tumors are of epithelial origin. ${ }^{192} 3637$ According to international societies and guidelines, including NCCN, ESMO/ESGO/ESTRO, fertilitysparing surgery is based on unilateral salpingooophorectomy and complete surgical staging with preservation of the uterus and contralateral ovary. This is possible for most young women who desire future fertility and have any stage malignant germ cell tumors, sex-cord stromal tumors, borderline tumors, or early stage epithelial carcinomas. ${ }^{1920}$ The key point is to avoid rupture of the mass. ${ }^{38}$

Among the literature on ovarian cancer and fertility-sparing options, we have selected two articles (a descriptive study ${ }^{6}$ and a narrative review ${ }^{5}$ on MRI); two studies (a prospective study ${ }^{39}$ and a 

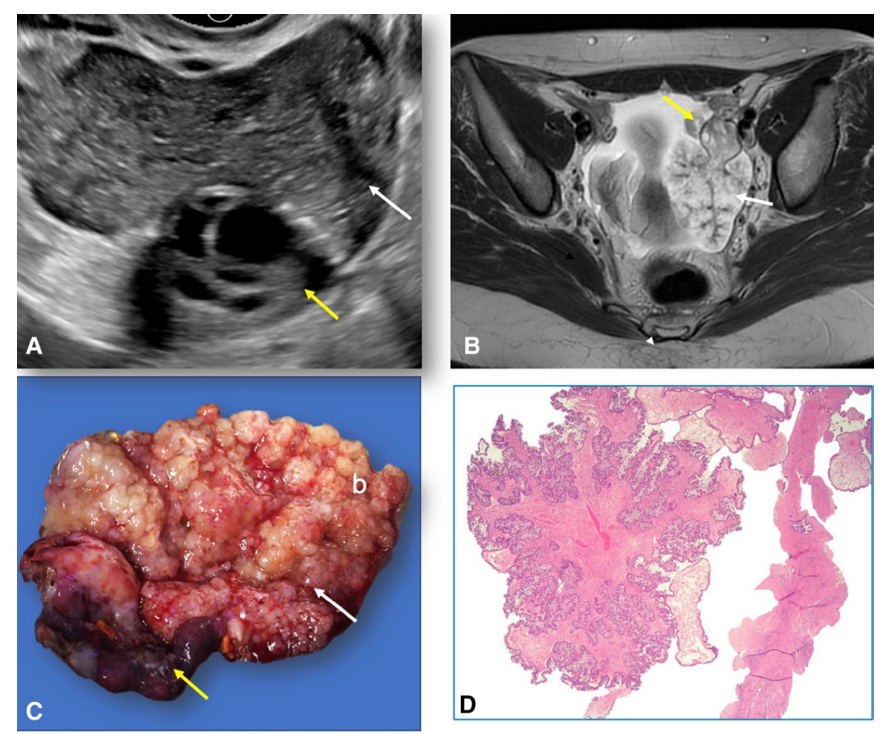

Figure 6 Ultrasound, MRI, and macroscopic images of a 29 years' old patient who underwent a left salpingoopherectomy for a left adnexal mass. Gray-scale ultrasound images (a) shows a left normal ovary (yellow arrow) and an exophytic mass (white arrow). Axial T2-weighted MR image (b) shows the left ovary (yellow arrow) with an exophytic mass (white arrow) with multiple nodular papillar projections with restricted diffusion at diffusion-weighted MR imaging and a type t2 curve (not shown). Macroscopy (c) confirmed the left exophytic tumor (white arrow) adjacent to normal ovary (yellow arrow). Final histology (d) was positive for serous borderline tumor with microinvasive foci.

narrative review ${ }^{4}$ on ultrasound evaluation); a prospective study ${ }^{40}$ on intraoperative ultrasound: and finally, one narrative review ${ }^{7}$ on fertility-sparing surgery and gynecological cancer, including data on MRI and ultrasound evaluation in ovarian cancer patients (Table 1). According to these articles, the eligibility criteria of fertility-sparing treatment on MRI and ultrasound should be based on the absence of extraovarian disease in young patients with ovarian masses suspicious for malignancy (borderline or invasive). ${ }^{45}$

The criteria for distinguishing between benign and malignant adnexal masses on $\mathrm{MRI}^{5-7}$ and ultrasound ${ }^{41}$ have been extensively described in the literature.

\section{Ultrasound Methodology}

Ovarian lesions are described as ultrasound according to International Ovarian Tumor Analysis (IOTA) terminology. ${ }^{41}$ In particular, ovarian tumor is measured in three diameters, in two perpendicular planes. The morphology aspect of the mass, cystic content, external and internal walls, presence of solid component, as well as of papillary projections, septum, and vascularization are reported (Figure 6). ${ }^{41}$ Based on pattern recognition in transvaginal examination, an expirienced examiner is able to provide high accuracy in selecting malignant lesions. ${ }^{42}$

The IOTA ADNEX model has been now extensively validated providing a high accuracy in discriminating between benign and malignant adnexal masses. ${ }^{43}$ This model is also able to provide the relative risk of each subgroups of malignancy (borderline, early stage, advanced, and metastatic tumors). However, the discrimination between borderline and invasive early-stage ovarian cancer, which is clinically relevant in planning the correct surgical treatment in young patients, remains a clinical challenge. ${ }^{44} 45$ Transvaginal examination is now able to identity ovarian lesions of very small size, thus representing a significant role in the follow-up of patients after fertilitysparing surgery. ${ }^{39} \mathrm{~A}$ large series of young women previously treated with fertility-sparing surgery and presenting with ovarian recurrences have been followed for a long period with transvaginal examination. It has been demonstrated that borderline ovarian recurrences can be detected at a very small size and the growth rate is about $1-2 \mathrm{~mm} /$ month. These findings are clinically useful in planning surveillance of these patients in order to plan a subsequent fertility-sparing surgery at the most appropriate time (ie, when the ovarian recurrence that had been detected in the inner part of the ovary is visible at the surface of the ovary). ${ }^{39}$ In some patients, an intraoperative ultrasound examination facilitated complete tumor excision in recurrent ovarian borderline tumors minimizing the removal of ovarian tissue. ${ }^{404647}$ Regarding the preoperative assess of extraovarian disease, ultrasound examination demonstrated high specificity but low sensitivity in the detection of abdominal peritoneal carcinomatosis. ${ }^{48-50}$

\section{MRI Protocol and Methodology}

The MRI protocol for the evaluation of patients with ovarian cancer is similar to the previous one described for cervical cancer but with some differences. High-resolution T2-weighted sequences are performed in the sagittal, axial, and axial oblique T2WI planes. The axial oblique plane is obtained being parallel to the endometrial cavity (referred as "ovarian axis"), which can assist in visualizing the gonadal vessels to confirm the ovarian origin of the mass. Axial diffusion-weighted imaging is used for adnexal mass assessment ( $b=0$ and $800-1000 \mathrm{sec} / \mathrm{mm}^{2}$ ) and apparent diffusion coefficient maps are obtained. Diffusionweighted imaging should always be evaluated in conjunction with other sequences such as T2WI and contrast-enhanced images. It is fundamental for adnexal masses because both benign and malignant ovarian masses may show diffusion restriction (high-signal intensity on high b-value images and low-signal intensity on apparent diffusion coefficient maps).

DCE-MRI is acquired on the axial plane applying a dynamic multiphase approach or perfusion imaging. Perfusion imaging is performed for a minimum of $3 \mathrm{~min}$ after contrast administration with a temporal resolution less than $15 \mathrm{~s}$. Perfusion images are evaluated by placing regions of interest on the most avidly enhancing portion of the ovarian mass and on the outer myometrium determining one of three types of time-intensity curves (using the enhancement of the outer myometrium as a reference). The Type 1 curve demonstrates a low level of enhancement without plateau, and indicates benignity. The type 3 curve, rapid high level of enhancement (faster than that of the myometrium), suggests possible malignancy. An in-between Type 2 curve, moderate level of enhancement with a plateau, may indicate for borderline ovarian tumor.

$\mathrm{MRI}$ characteristics that are highly predictive of benign disease include purely cystic morphology, cystic morphology with blood content as endometriomas, and purely fatty masses; absence of wall enhancement; and masses with solid components with low T2 signal intensity. ${ }^{7}$ When a solid enhancing mass is present a slow wash-in or a time-signal-intensity curve with slow gradual enhancement (Type 1 curve) is predictive of benign lesions. ${ }^{7}$ MRI 
characteristics indicative of malignant masses (Figure 6) are represented by complex solid cystic lesions whose solid components have intermediate T2 signal intensity and, when compared with the adjacent myometrium, demonstrating a rapid wash-in rate and more intense enhancement during dynamic contrast-enhanced MR imaging (Type 3 curve). ${ }^{7}$ However, an algorithmic evaluation of the adnexal mass can be used to determine the associated risk for malignancy (ADNEX MR Imaging Score). ${ }^{71}$

MRI reports to assess eligibility for fertility-sparing treatment in patients with ovarian cancer include:

i. Tumor information: evaluation of adnexal mass with description of risk of malignancy, as described above.

ii. Other ovarian information: presence of preserved ipsilateral ovarian and evaluation of contralateral ovary.

iii. Lymph nodes information: absence or presence of pelvic or para-aortic nodal enlargement (short-axis diameter larger than $10 \mathrm{~mm}$ ). Other suspicious lymph nodes characteristics consist of round morphology, irregular borders, and presence of necrosis.

iv. Other findings in pelvis or abdomen: presence of peritoneal implants, bladder, rectum, liver, kidney, bone.

Computer tomography (CT) staging. When a mass is suspected of being malignant on the basis of ultrasound examination, the detection of additional localization of disease in the pelvis and peritoneum becomes important for the detection of measurable disease beyond the mass, which would change the fertility preservation decision. CT is the current standard technique for this detection, as suggested by the National Institute for Health and Care Excellence, the European Society of Urogenital Radiology, and the American College of Radiology. ${ }^{12}$ However, CT has low sensitivity for the identification of small peritoneal nodules and unsatisfactory accuracy in the staging of early peritoneal disease.

\section{DISCUSSION}

Ultrasound and MRI play a key role in selecting patients who might benefit from fertility preservation in cervical and endometrial cancers. There is also evidence supporting the fundamental role of both ultrasound and MRI in identifying adnexal masses, however, their efficacy in excluding metastatic disease outside the ovary in patients with ovarian cancers remains to be clarified. We recognize that our study is a narrative review and not a systematic review or metanalysis, lacking important requirements for quality control such as risk of bias, quality assessment, and statistical analysis. Our goal was to provide an overview of the literature on imaging and fertility preservation, and to offer parameters on the management of these patients in clinical practice.

Other reviews have focused on imaging and fertility-sparing surgery, however, most analyzed only data on MRI and cervical cancer, ${ }^{810}$ or MRI and gynecological cancers, ${ }^{5}$ and only one narrative review reported data on ultrasound and gynecological cancers. ${ }^{4}$ In particular, Rob et al, ${ }^{8}$ in a narrative review, evaluated the selection criteria based on MRI for the preservation of fertility in cervical cancer, but the authors manly focused on the surgical procedures of fertility-sparing rather than imaging data. De Boera et $\mathrm{al}^{10}$ conducted a systematic review on the accuracy of MRI in detecting involvement of the internal uterine os in cervical cancer patients, without providing which parameters are needed for selecting patients for fertility preservation. The authors found that MRI had a sensitivity of $91 \%$ and specificity of $97 \%$ in evaluating involvement of the internal os, using histology as a reference standard. Similar to ourstudy, McEvoy et al ${ }^{5}$ reported a narrative review on the role of imaging in the proper selection of patients with gynecological cancers for fertility preservation, but in that study the authors focused only on MRI. They found that MRI criteria were tumor size $\leq 2 \mathrm{~cm}$, tumor-to-internal os distance $\geq 1 \mathrm{~cm}$ (or alternatively $\geq 0.5 \mathrm{~cm}$ ), and cervical stromal invasion $<50 \%$, for cervical cancer: disease confined to the endometrium with the absence of any myometrial invasion and stromal invasion, absence of synchronous primary ovarian cancer, or ovarian metastases, for endometrial cancer. However, the authors did not report any specific indication for fertility preservation in ovarian cancer patients but only suggested that MRI is useful in discriminating between benign and malignant tumors. ${ }^{5}$ Fischerova et $\mathrm{al}^{4}$ described the ultrasound methodology of gynecological cancer staging, providing ultrasound criteria for selecting patients for fertility-sparing surgery. However, the authors were mainly based on methodology and sonographic features, with some clinical considerations in assessing local tumor extent, lymph nodes, and distant metastases by ultrasound examination.

In conclusion, fertility preservation is an integral component of clinical decision-making and treatment planning. Therefore, knowledge of the imaging parameters that guide clinicians in selecting patients who are candidates for fertility preservation is of the utmost importance. This review provides an update of the data used for the counseling and management of patients with a desire for fertility preservation. This study may provide an insight into areas that still need to be prospectively explored and may provide new perspectives for further multicenter studies.

\section{Author affiliations \\ ${ }^{1}$ Dipartimento Scienze della Salute della Donna, del Bambino e di Sanità Pubblica, Fondazione Policlinico Universitario Agostino Gemelli, IRCCS, Rome, Italy \\ 2Dipartimento di Diagnostica per Immagini, Radioterapia Oncologica ed Ematologia, Fondazione Policlinico Universitario Agostino Gemelli, IRCCS, Rome, Italy \\ ${ }^{3}$ Dipartimento Scienze della vita e di Sanità Pubblica, Università Cattolica del Sacro Cuore, Rome, Italy \\ ${ }^{4}$ Centro di Ricerca e Studi sulla Salute Procreativa, Università Cattolica del Sacro Cuore, Rome, Italy}

Acknowledgements We acknowledge Professor Gian Franco Zannoni and Dr Damiano Arciuolo, Fondazione Policlinico Universitario Agostino Gemelli, IRCCS, Rome, for macroscopic and microscopic images of clinical cases.

Contributors All authors contributed in the creation of the work.

Funding The authors have not declared a specific grant for this research from any funding agency in the public, commercial, or not-for-profit sectors.

Competing interests None declared.

Patient consent for publication Not required.

Provenance and peer review Not commissioned; externally peer reviewed.

ORCID iD

Francesca Moro http://orcid.org/0000-0002-5070-7245

\section{REFERENCES}

1 Woodruff TK. The Oncofertility Consortium - addressing fertility in young people with cancer. Nat Rev Clin Oncol 2010;7:466-75.

2 Carter J, Rowland K, Chi D, et al. Gynecologic cancer treatment and the impact of cancer-related infertility. Gynecol Oncol 2005;97:90-5.

3 Loren AW, Mangu PB, Beck LN, et al. Fertility preservation for patients with cancer: American Society of Clinical Oncology clinical practice guideline update. J Clin Oncol 2013;31:2500-10. 
4 Fischerova D. Ultrasound scanning of the pelvis and abdomen for staging of gynecological tumors: a review. Ultrasound Obstet Gynecol 2011;38:246-66.

5 McEvoy SH, Nougaret S, Abu-Rustum NR, et al. Fertility-sparing for young patients with gynecologic cancer: how MRI can guide patient selection prior to conservative management. Abdom Radiol 2017:42:2488-512.

6 Stein EB, Hansen JM, Maturen KE. Fertility-sparing approaches in gynecologic oncology: role of imaging in treatment planning. Radiol Clin North Am 2020;58:401-12.

7 Rockall AG, Qureshi M, Papadopoulou I, et al. Role of imaging in fertility-sparing treatment of gynecologic malignancies. Radiographics 2016;36:2214-33.

8 Rob L, Pluta M, Skapa P, et al. Advances in fertility-sparing surgery for cervical cancer. Expert Rev Anticancer Ther 2010;10:1101-14.

9 Rodolakis A, Biliatis I, Morice P, et al. European Society of Gynecological Oncology task force for fertility preservation: clinical recommendations for fertility-sparing management in young endometrial cancer patients. Int J Gynecol Cancer 2015;25:1258-65.

10 de Boer P, Adam JA, Buist MR, et al. Role of MRI in detecting involvement of the uterine internal os in uterine cervical cancer: systematic review of diagnostic test accuracy. Eur J Radiol 2013;82:e422-8.

11 Jumelle C, Leblanc E, Ceugnart L, et al. MR imaging in the management of trachelectomy. Diagn Interv Imaging 2016;97:129-32.

12 Lakhman Y, Akin O, Park KJ, et al. Stage IB1 cervical cancer: role of preoperative MR imaging in selection of patients for fertility-sparing radical trachelectomy. Radiology 2013;269:149-58.

13 Bhosale PR, Iyer RB, Ramalingam P, et al. Is MRI helpful in assessing the distance of the tumour from the internal os in patients with cervical cancer below FIGO Stage IB2? Clin Radiol 2016;71:515-22.

14 Noël P, Dubé M, Plante M, et al. Early cervical carcinoma and fertility-sparing treatment options: MR imaging as a tool in patient selection and a follow-up modality. RadioGraphics 2014;34:1099-119.

15 Marth C, Landoni F, Mahner S, et al. Cervical cancer: ESMO clinical practice guidelines for diagnosis, treatment and follow-up. Ann Oncol 2017;28:iv72-83.

16 Koh W-J, Abu-Rustum NR, Bean S, et al. Cervical cancer, version 3.2019 , NCCN clinical practice guidelines in oncology. J Natl Compr Canc Netw 2019;17:64-84.

17 Colombo N, Creutzberg C, Amant F, et al. ESMO-ESGO-ESTRO consensus conference on endometrial cancer: diagnosis, treatment and follow-up. Ann Oncol 2016;27:16-41.

18 Koh W-J, Abu-Rustum NR, Bean S, et al. Uterine neoplasms, version 1.2018, NCCN clinical practice guidelines in oncology. J Natl Compr Canc Netw 2018;16:170-99.

19 Morgan RJ, Armstrong DK, Alvarez RD, et al. Ovarian cancer, version 1.2016, NCCN clinical practice guidelines in oncology. J Natl Compr Canc Netw 2016;14:1134-63.

20 Ray-Coquard I, Morice P, Lorusso D, et al. Non-epithelial ovarian cancer: ESMO clinical practice guidelines for diagnosis, treatment and follow-up. Ann Oncol 2018;29:iv1-18.

21 Torre LA, Bray F, Siegel RL, et al. Global cancer statistics, 2012. CA Cancer J Clin 2015;65:87-108.

22 Peppercorn PD, Jeyarajah AR, Woolas R, et al. Role of MR imaging in the selection of patients with early cervical carcinoma for fertilitypreserving surgery: initial experience. Radiology 1999;212:395-9.

23 Downey K, Shepherd JH, Attygalle AD, et al. Preoperative imaging in patients undergoing trachelectomy for cervical cancer: validation of a combined T2- and diffusion-weighted endovaginal MRI technique at 3.0 T. Gynecol Oncol 2014;133:326-32.

24 Pálsdóttir K, Fischerova D, Franchi D, et al. Preoperative prediction of lymph node metastasis and deep stromal invasion in women with invasive cervical cancer: prospective multicenter study using 2D and 3D ultrasound. Ultrasound Obstet Gynecol 2015;45:470-5.

25 Testa AC, Di Legge A, De Blasis I, et al. Imaging techniques for the evaluation of cervical cancer. Best Pract Res Clin Obstet Gynaecol 2014;28:741-68.

26 Haldorsen IS, Lura N, Blaakær J, et al. What is the role of imaging at primary diagnostic work-up in uterine cervical cancer? Curr Oncol Rep 2019;21:77:1-15.

27 WHO. GLOBOCAN 2012: estimated cancer incidence, mortality and prevalence worldwide in 2012, 2012. Available: http://globocan.iarc. fr/Pages/fact sheets population.aspx [Accessed 3 Apr 2015].

28 Cade TJ, Quinn MA, McNally OM, et al. Predictive value of magnetic resonance imaging in assessing myometrial invasion in endometrial cancer: is radiological staging sufficient for planning conservative treatment? Int J Gynecol Cancer 2010;20:1166-9.
29 Suh D-S, Kim JK, Kim KR, et al. Reliability of magnetic resonance imaging in assessing myometrial invasion absence in endometrial carcinoma. Acta Obstet Gynecol Scand 2009;88:990-3.

30 Sakane M, Hori M, Onishi H, et al. Assessment of myometrial invasion in premenopausal grade 1 endometrial carcinoma: is magnetic resonance imaging a reliable tool in selecting patients for fertility-preserving therapy? J Comput Assist Tomogr 2018;42:412-7.

31 Rodolakis A, Biliatis I, Morice P, et al. European Society of Gynecological Oncology task force for fertility preservation: clinical recommendations for fertility-sparing management in young endometrial cancer patients. Int $J$ Gynecol Cancer 2015;25:1258-65.

32 Leone FPG, Timmerman D, Bourne T, et al. Terms, definitions and measurements to describe the sonographic features of the endometrium and intrauterine lesions: a consensus opinion from the International Endometrial Tumor Analysis (IETA) group. Ultrasound Obstet Gynecol 2010;35:103-12.

33 Epstein E, Fischerova D, Valentin L, et al. Ultrasound characteristics of endometrial cancer as defined by International Endometrial Tumor Analysis (IETA) consensus nomenclature: prospective multicenter study. Ultrasound Obstet Gynecol 2018;51:818-28.

34 Mascilini F, Testa AC, Van Holsbeke C, et al. Evaluating myometrial and cervical invasion in women with endometrial cancer: comparing subjective assessment with objective measurement techniques. Ultrasound Obstet Gynecol 2013:42:353-8.

35 Savelli L, Ceccarini M, Ludovisi M, et al. Preoperative local staging of endometrial cancer: transvaginal sonography vs. magnetic resonance imaging. Ultrasound Obstet Gynecol 2008;31:560-6.

36 Colombo N, Parma G, Zanagnolo V, et al. Management of ovarian stromal cell tumors. J Clin Oncol 2007;25:2944-51.

37 Satoh T, Hatae M, Watanabe Y, et al. Outcomes of fertility-sparing surgery for stage I epithelial ovarian cancer: a proposal for patient selection. J Clin Oncol 2010;28:1727-32.

38 Vergote I. Role of surgery in ovarian cancer: an update. Acta Chir Belg 2004;104:246-56.

39 Franchi D, Boveri S, Radice D, et al. Ultrasonographic diagnosis and longitudinal follow-up of recurrences after conservative surgery for borderline ovarian tumors. Am J Obstet Gynecol 2016;215:756. e1-756.e9.

40 Jones BP, Saso S, Farren J, et al. Ultrasound-guided laparoscopic ovarian wedge resection in recurrent serous borderline ovarian tumours. Int J Gynecol Cancer 2017;27:1813-8.

41 Timmerman D, Valentin L, Bourne TH, et al. Terms, definitions and measurements to describe the sonographic features of adnexal tumors: a consensus opinion from the International Ovarian Tumor Analysis (IOTA) group. Ultrasound Obstet Gynecol 2000;16:500-5.

42 Valentin L. Pattern recognition of pelvic masses by gray-scale ultrasound imaging: the contribution of Doppler ultrasound. Ultrasound Obstet Gynecol 1999;14:338-47.

43 Van Calster B, Valentin L, Froyman W, et al. Validation of models to diagnose ovarian cancer in patients managed surgically or conservatively: multicentre cohort study. BMJ 2020;370:m2614.

44 Fagotti A, Ludovisi M, De Blasis I, et al. The sonographic prediction of invasive carcinoma in unilocular-solid ovarian cysts in premenopausal patients: a pilot study. Hum Reprod 2012;27:2676-83.

45 Moro F, Baima Poma C, Zannoni GF, et al. Imaging in gynecological disease (12): clinical and ultrasound features of invasive and non-invasive malignant serous ovarian tumors. Ultrasound Obstet Gynecol 2017:50:788-99.

46 Mascilini F, Quagliozzi L, Bolomini G, et al. Intraoperative ultrasound through laparoscopic probe in fertility-sparing surgery for borderline ovarian tumor recurrence. Ultrasound Obstet Gynecol 2019;54:280-2.

47 De Blasis I, Tortorella L, Macchi C, et al. Intraoperative ultrasound diagnosis of metastatic lymph node in serous borderline ovarian tumor. Ultrasound Obstet Gynecol 2019;54:562-3.

48 Fischerova D, Zikan M, Semeradova I, et al. Ultrasound in preoperative assessment of pelvic and abdominal spread in patients with ovarian cancer: a prospective study. Ultrasound Obstet Gynecol 2017;49:263-74.

49 De Blasis I, Moruzzi MC, Moro F, et al. Role of ultrasound in advanced peritoneal malignancies. Minerva Med 2019;110:292-300.

50 Fischerová D, Pinto P, Kocian R, et al. OC14.06: preoperative staging of advanced ovarian cancer: comparison between ultrasound, CT and WB-DWI/MRI. Ultrasound Obstet Gynecol 2017;50.

51 Thomassin-Naggara I, Rockall A. Editorial for "Validity and Reproducibility of ADNEX MR SCORING System in Diagnosis of Sonographically Indeterminate Adnexal Masses". J Magn Reson Imaging 2020;21:1-2. 